Interfaces and Free Boundaries 12 (2010), 443-462

DOI $10.4171 / \mathrm{IFB} / 241$

\title{
The Neumann problem in an irregular domain
}

\author{
ŁUKASZ BolikowsKi, MARIA GOKIELI, NiCOLAS VARCHON \\ Interdisciplinary Centre for Mathematical and Computational Modelling, \\ University of Warsaw \\ Żwirki i Wigury 93, 02-089 Warszawa, Poland \\ E-mail: L.Bolikowski@icm.edu.pl,M.Gokieli@icm.edu.pl,N.Varchon@icm.edu.pl
}

[Received 29 November 2008 and in revised form 16 April 2010]

\begin{abstract}
We consider the stability of patterns for the reaction-diffusion equation with Neumann boundary conditions in an irregular domain in $\mathbb{R}^{N}, N \geqslant 2$, the model example being two convex regions connected by a small 'hole' in their boundaries. By patterns we mean solutions having an interface, i.e. a transition layer between two constants. It is well known that in 1D domains and in many 2D domains, patterns are unstable for this equation. We show that, unlike the $1 \mathrm{D}$ case, but as in $2 \mathrm{D}$ dumbbell domains, stable patterns exist. In a more general way, we prove invariance of stability properties for steady states when a sequence of domains $\Omega_{n}$ converges to our limit domain $\Omega$ in the sense of Mosco. We illustrate the theoretical results by numerical simulations of evolving and persisting interfaces.
\end{abstract}

\section{Introduction}

Let us consider the Neumann boundary problem for the reaction-diffusion equation

$$
u_{t}-\Delta u=g(u)
$$

on domains which have a crack, or 'splitting' inside: see Figure 1 .

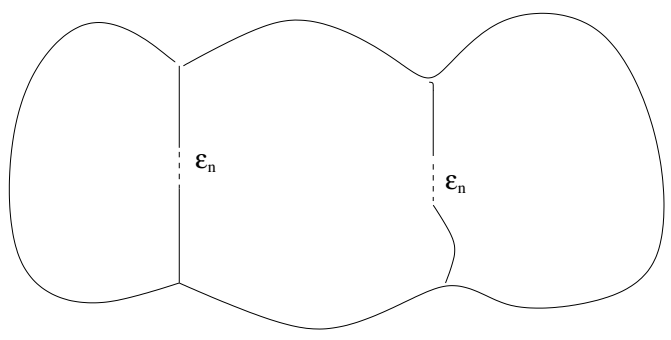

FIG. 1. Our model domain $\Omega_{n}$.

We are particularly interested in the steady, i.e. independent of time, solutions of (1), which we will also call equilibria or stationary states. Indeed, as part of the attractor, the steady states determine to a very large extent the evolution of any initial data. With respect to this process, the main feature of a steady solution is its stability, that is, roughly speaking, its being or not 'attractive' for other solutions in some neighborhood. This stability is expressed by the sign of the eigenvalues for the linearized problem. 
It is well known that stability is strongly influenced by the domain's shape. First of all, for Neumann boundary conditions and convex regions $\Omega$, the only stable solutions are constants (given by the zeros of $g$ ). This has been proved in 1978 by Casten and Holland [CH] and independently in 1979 by Matano $[\mathrm{M}]$. It is also shown in $[\mathrm{CH}]$ that this crucial property holds for a larger class of domains including annuli, and for all domains provided $g$ is convex. On the other hand, Matano [M] constructs an example of a connected region - of quite complex shape-for which there exist nonconstant stable equilibria: they are nearly constant in some parts of the domain but possess also interfaces between these constant parts. In other words, he shows existence of a domain for which 'pattern formation' occurs for the reaction-diffusion equation.

Can this be expected for simpler shapes? Many works (Hale and Vegas [HV], Vegas [V], Jimbo [J1, J2], Jimbo and Morita [JM, MJ], de Oliveira et al. [OPP], Arrieta et al. [ACL] and references to previous works therein) addressed this question for dumbbell domains: two bigger regions connected by a thin strip. The answer, under various assumptions, is positive: stable interfaces exist. We want to ask here if the same occurs for 'split' regions, with cracks inside, as the one in Figure 1 . We state below the conditions defining the class of our admissible domains.

Let us start by presenting some numerical experiments so as to illustrate the idea of this work. For the experiments, we have taken the example of the Allen-Cahn nonlinearity:

$$
g(u)=u\left(1-u^{2}\right),
$$

so that (1) has two stable equilibria, 1 and -1 , and one unstable, equal to 0 . Figure 2 shows the results of three simulations. (We refer to the Appendix, Section 5 for more details on the numerical method used here and for more investigations on the dynamics.)

(a)
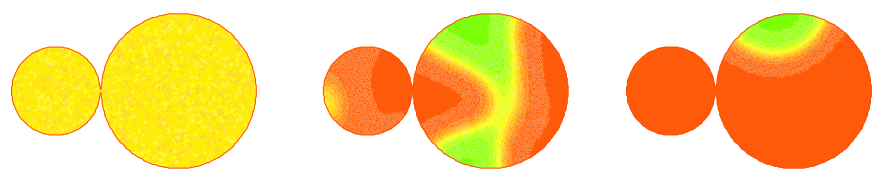

(b)
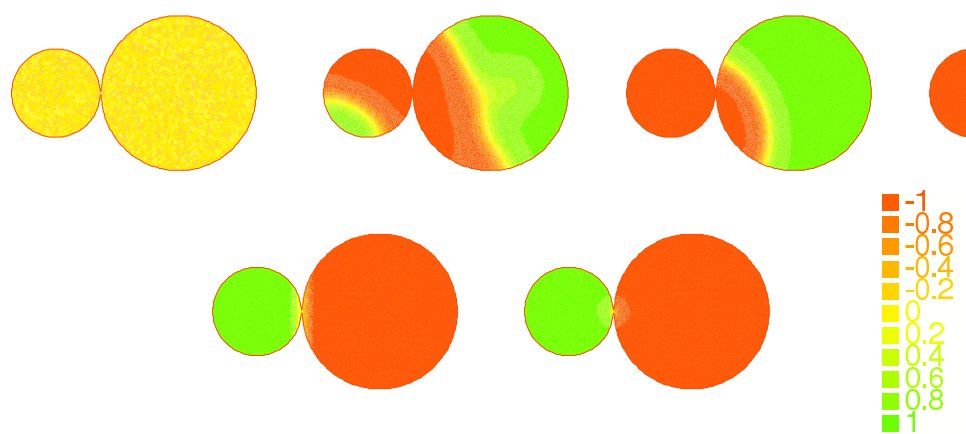
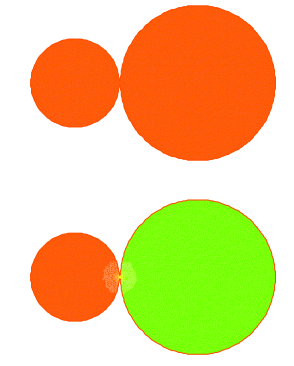

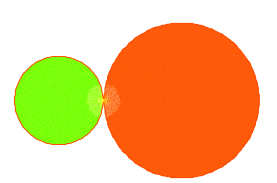

(c)

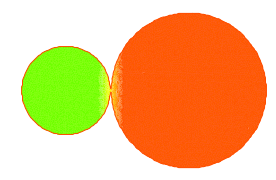

FIG. 2. (a) The initial data $u_{0} \approx 0$, with a random perturbation, on $\Omega_{n}$. We visualize the evolution at $t=0, t=10$, $t=40$ and $t=100$. The last state is constant. (b) The same experiment, the initial data $u_{0}=0$ being again randomly perturbed; $t=0, t=10, t=100$ and $t=200$. The last state has an interface at the level of the connection between the two subdomains. (c) $u_{0} \approx 1$ on the left, $u_{0} \approx-1$ on the right, $t=0$ and $t=1400$. The state possessing an interface seems not to evolve.

Experiments (a) and (b) are performed with initial data taken as a random perturbation of the constant 0 , the stationary unstable solution. The system evolves towards two different states: 
a constant solution (equal to -1 ) in case (a) and a nonconstant one, close to \pm 1 in each subregion in case (b). It is clear that the first one is stable. The experiment (c) is performed so as to verify the stability of the nonconstant steady state. We take there an initial datum equal to 1 in the domain on the left-hand side part of $\Omega_{n}$ and equal to -1 on the right-hand side part, except for a transition layer in a neighborhood of the connection, where it is linearly interpolated. This datum does not seem to evolve towards a constant state.

This visual impression is in no way a proof of any stability, not only because the domain is approximated, but even more in view of the known results about the extreme slowness of the evolution of the Allen-Cahn equation and of the 'dormant instability' of an analogous, nonconstant and monotone solution on a segment in one dimension (see Fusco and Hale $[\overrightarrow{F H}]$ ). However, as we prove below, our two-dimensional geometry ensures the stability of this solution.

We actually prove the continuity of the stability properties of each steady stable or unstable (hyperbolic) solution with respect to domain perturbations. For many usual nonlinearities $g$ having no degenerate zeros, we are able to count all steady solutions.

We will deal with the problem by considering it as a perturbation of a limit problem, posed on a set $\Omega$ which will typically be disconnected, but such that $\bar{\Omega}$ is connected. Also the domains $\Omega_{n}$ that we want to consider are not regular, their boundaries are not locally graphs of functions and they do not admit continuous extension operators $E: H^{1}(\omega) \rightarrow H^{1}\left(\mathbb{R}^{n}\right), \omega=\Omega$ or $\Omega_{n}$. In this regard our work complements the paper of Arrieta and Carvalho $[\mathrm{AC}]$, which deals with the same problem for regular domains with Lipschitz boundaries.

Let us list our main assumptions on $\Omega$ and its admissible perturbations and comment on their concrete realizations.

$\left(C_{1}\right) \Omega$ is an open set, $\left(\Omega_{n}\right)_{n \in \mathbb{N}}$ a sequence of open sets and $D$ a ball in $\mathbb{R}^{n}$ such that

$$
\forall n \in \mathbb{N}, \quad \Omega \subset \Omega_{n+1} \subset \Omega_{n} \subset D ;
$$

$\left(C_{2}\right)\left|\Omega_{n}\right|=|\Omega|$ for all $n \in \mathbb{N}$;

$\left(C_{3}\right)$ the injection from $H^{1}(\Omega)$ into $L^{2}(\Omega)$ is compact;

$\left(C_{4}\right)\left(\Omega_{n}\right)$ converges in the sense of Mosco to $\Omega$.

We also assume that $g$ is a $C^{1}(\mathbb{R})$ function satisfying

(G) $\limsup _{|x| \rightarrow+\infty} \frac{g(x)}{x}<0$.

The geometrical sense of $\left(C_{1}\right)$ and $\left(C_{2}\right)$ is clear. $\left(C_{3}\right)$ is a condition on $\partial \Omega$ 's regularity, but it is very weak - enough to allow splittings and disconnected $\Omega$. Indeed, note first that if $\Omega$ has a finite number of connected components, the compactness of the injection holds for $\Omega$ if it holds for every connected component of $\Omega$. On the other hand, as far as connected sets are concerned, it is satisfied by domains having the cone property, and, more generally, by unions of a finite number of domains admitting an extension operator continuous in the $H^{1}$-norm. See the book of Maz'ya [Maz, Sections 1.6 and 1.10] for a review of results on this point and for examples. We give a nonstandard example of an admissible $\Omega$ in Figure 3 (cf. [Maz, 1.5, p. 38]).

Let us comment on $\left(C_{4}\right)$. The definition of Mosco convergence is introduced rigorously in Section 2 Its essential feature is to be equivalent to convergence of solutions of the stationary Neumann problem on $\Omega_{n}$ to the solution of this problem on $\Omega$. This holds independently of the space dimension (see the paper of Dal Maso et al. [DMa] for the nonlinear setting and references 


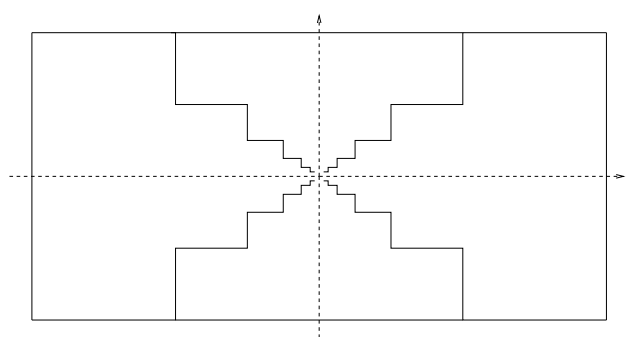

FIG. 3. An admissible planar set $\Omega$ : the rectangle $(-2,2) \times(-1,1)$ deprived of four polylines: $\left\{(x, y): 2^{-k}<|x|<2^{1-k}\right.$, $|y|=2^{-k}$ or $\left.|x|=2^{-k}, 2^{-k-1}<|y|<2^{-k}, k=0,1, \ldots\right\}$.

therein for the linear one; the linear problem should be formulated in quotient spaces so as to have uniqueness). So, the use of Mosco convergence seems the most appropriate and general approach to our problem. However, as its definition is not geometrical, it is important to confront it with more intuitive notions of set convergence. Indeed, for planar domains $\Omega_{n}$ having a bounded number of 'holes' (connected components of $\Omega_{n}^{c}$ ) and converging to $\Omega$ in the Hausdorff complementary topology, it has been shown by Bucur and Varchon in [BV] that Mosco convergence is equivalent to the condition meas $\left(\Omega_{n}\right) \rightarrow$ meas $(\Omega)$. This gives, directly, a very wide range of domains in $\mathbb{R}^{2}$ for which our results apply. In higher dimensions, Mosco convergence is more difficult to obtain; see e.g. Cortesani [Cor] who shows unstability of the linear Neumann problems satisfying the assumptions of [BV]; cf. also Damlamian [Dam] and many other works related to the Neumann sieve. However, it is known (see for instance Henrot [H1]) that Mosco convergence occurs if the capacity of $\Omega_{n} \backslash \Omega$ converges to 0 . (By capacity we mean here the 2-capacity; we refer to Evans and Gariepy [EG, Chapter 4] for the notion and properties of capacity, which is a tool for measuring very fine sets. Let us just note here that all sets in $\mathbb{R}^{N}$ of Hausdorff dimension greater than $N-2$ are of nonzero capacity.) Thus, one can see that $\left(C_{4}\right)$ is satisfied for example if the connected components of $\Omega$ are each at zero distance from some other, and $\Omega_{n}$ is obtained by 'making holes' in the joining parts of the boundary, under the condition that the number of holes does not grow too rapidly and their sizes diminish. For most applications it is sufficient to assume that the number of holes remains constant.

The plan of this work is as follows. We begin by giving the main mathematical tools (Section 2), then state the main theorems on stability (Section 3) and finally we study convergence of the evolution problem (Section 4). In the main part, we show in Theorem 3.4, under the assumptions above, that any hyperbolic, i.e. linearly stable or unstable steady state on $\Omega$ is a limit of a sequence $\left(u_{n}\right)$ of hyperbolic steady states on $\Omega_{n}$, in the sense of $L^{2}(D)$ convergence. In Theorem 3.5. we show that, moreover, for $n$ large enough, $u_{n}$ has the same stability as $u$ : the eigenvalues of the linearized operator $-\Delta-g^{\prime}\left(u_{n}\right)$ converge to eigenvalues of the operator $-\Delta-g^{\prime}(u)$ (where $\Delta$ means the Neumann-Laplacian and the second term is a multiplication operator). We also have convergence of all respective eigenspaces, still in the sense of $L^{2}(D)$ distance (between unit eigenvectors).

Existence of nonconstant stable equilibria on $\Omega_{n}$, for large $n$, is an immediate consequence of Theorems 3.4 and 3.5. Indeed, take $g$ with two stable zeros, like $g(u)=u\left(1-u^{2}\right)$, and set $u$ equal to +1 and -1 on each connected component of $\Omega$. Then $u$ forms a stable equilibrium which by our result can be approximated, in $L^{2}(D)$, by a sequence $u_{n}$ of stable equilibria on $\Omega_{n}$. What remains an open question is the rate of this convergence: how small the 'hole' must be for the equilibria to become stable. 
In the case when the system admits only hyperbolic equilibria, we show in Theorem 3.8 that their number is equal on $\Omega$ and on $\Omega_{n}$. Theorem 3.9 states that in this case, the Hausdorff distance in $L^{2}(D)$ between the sets of stable steady points on $\Omega_{n}$ and the set of stable steady points on $\Omega$ goes to zero. The same is true for unstable equilibria.

Our method is based on the degree and operator perturbation theories. The perturbation analysis is very close to the one performed in $[\overline{\mathrm{AC}}]$, and the degree theory (the Leray-Schauder fixed point index) that we use in Theorem 3.4 has also been applied by the same authors in [ACL] for dumbbell domains. Also, the results that we obtain have their analogues in [AC, Propositions 3.1, 4.1 and Corollary 4.3]. In [AC], continuity of unstable manifolds, and thus attractors, is also proved. In this point, however, the authors rely strongly on the existence of an extension operator continuous in $H^{1}$, which does not exist for our model domains. (Note that planar domains for which such an operator exists are known to be quasi-conformal to disks; see [Maz, Comments to Section 1.6] and references therein). Theorem 3.4 could also be deduced from the results of [DMa]; however, it seemed simpler to give a direct proof.

Finally, we devote Section 4 to the evolution problem. We estimate the difference of the semigroups in a norm containing an exponential weight with respect to time. This reflects the fact that, in spite of the results about steady states, the dynamics on $\Omega_{n}$ and on $\Omega$ are of course different.

\section{Preliminaries}

\subsection{Main operator, linear problem}

For all $f \in L^{2}(\Omega)$, the linear equation

$$
\begin{cases}-\Delta u+u=f & \text { in } \Omega, \\ \frac{\partial u}{\partial n}=0 & \text { on } \partial \Omega,\end{cases}
$$

has a unique variational solution in $H^{1}(\Omega)$.

Definition 2.1 We will denote by $A_{\Omega}$ the Neumann-Laplacian operator $-\Delta+I$,

$$
A_{\Omega}: L^{2}(\Omega) \rightarrow L^{2}(\Omega), \quad A_{\Omega} v=-\Delta_{N} v+v,
$$

considered in the domain

$$
D\left(A_{\Omega}\right)=\left\{u \in H^{1}(\Omega): \exists f \in L^{2}(\Omega) \text { such that } u \text { is the solution of }[2]\right\} .
$$

The operator $A_{\Omega}$ is selfadjoint, closed, has compact resolvent and its first eigenvalue is equal to 1 . A simple calculation using the canonical expansion in the basis of eigenvectors (cf. [H2, 1.3]) shows that $A_{\Omega}$ satisfies

$$
\left\|\left(\lambda-A_{\Omega}\right)^{-1}\right\|_{\mathcal{L}\left(L^{2}(\Omega)\right)} \leqslant \frac{2}{|\lambda-1|}
$$

for all $\lambda$ in the sector

$$
\mathcal{S}=\{\lambda: \pi / 3 \leqslant|\arg (\lambda-1)| \leqslant \pi, \lambda \neq 1\},
$$

i.e. it is sectorial in the sense of Henry [H2, Definition 1.3.1]. We have denoted by $\mathcal{L}\left(L^{2}(\Omega)\right)$ the space of continuous linear operators in $L^{2}(\Omega)$. The operator $-A_{\Omega}$ is then (see [H2, Theorem 1.3.4]) 
the infinitesimal generator of an analytic linear semigroup $\left\{S_{\Omega}(t)\right\}_{t} \geqslant 0$, which can be written as

$$
S_{\Omega}(t)=\frac{1}{2 i \pi} \int_{\Gamma} e^{\lambda t}\left(\lambda+A_{\Omega}\right)^{-1} \mathrm{~d} \lambda
$$

and $\Gamma$ is a contour in the resolvent set $\rho\left(-A_{\Omega}\right)$, with $\arg \lambda \rightarrow \pm \theta$ as $|\lambda| \rightarrow \infty$ for some $\theta \in$ $(\pi / 2, \pi)$. We also have (cf. [H2, Theorem 1.3.4])

$$
\left\|S_{\Omega}(t)\right\|_{\mathcal{L}\left(L^{2}(\Omega)\right)} \leqslant e^{-t}, \quad\left\|S_{\Omega}(t)\right\|_{\mathcal{L}\left(L^{2}(\Omega), H^{1}(\Omega)\right)} \leqslant \frac{e^{-t}}{\sqrt{t}}
$$

\subsection{Definition and basic properties of solutions}

For our analysis of the problem, we will now write (1) as

$$
\left\{\begin{array}{l}
u_{t}+A_{\Omega} u=f(u), \quad t>0 \\
u(0)=u_{0}
\end{array}\right.
$$

with

$$
f=g+\mathrm{Id} .
$$

Note that the assumption $(G)$ now takes the form

$$
\limsup _{|x| \rightarrow+\infty} \frac{f(x)}{x}<1
$$

DEFINITION 2.2 By a solution of (1) on an open set $\Omega$, with an initial condition $u_{0} \in L^{2}(\Omega)$, we understand a continuous function from $[0,+\infty)$ into $L^{2}(\Omega)$, satisfying on $(0,+\infty)$ the integral equation

$$
u(t)=S_{\Omega}(t) u_{0}+\int_{0}^{t} S_{\Omega}(t-s) f(u(s)) \mathrm{d} s
$$

with $S_{\Omega}$ given by (4). By a stationary solution (steady point, equilibrium) of (1) we mean a solution of

$$
A_{\Omega} u=f(u) .
$$

The set of stationary points will be denoted by $S P(\Omega)$.

Our notion of solution corresponds to what is often called a 'mild' solution. Every solution in the sense of Definition 2.2 satisfies (6); that is, mild solutions are also variational (weak) solutions. This is shown by calculating $u(t+h)-u(t)$ and passing to the limit $h \rightarrow 0$ (cf. [H2, Lemma 3.3.2]). Let us now consider the stationary states.

DEFINITION 2.3 The linearization of $A_{\Omega}-f$ at $u \in S P(\Omega)$ is the closed linear operator

$$
\begin{gathered}
A_{\Omega}-f^{\prime}(u): L^{2}(\Omega) \rightarrow L^{2}(\Omega), \\
\left(A_{\Omega}-f^{\prime}(u)\right) v=A_{\Omega} v-f^{\prime}(u) v, \\
D\left(A_{\Omega}-f^{\prime}(u)\right)=D\left(A_{\Omega}\right) .
\end{gathered}
$$


For all $u \in S P(\Omega)$ and $k \in \mathbb{N}$ let $\lambda_{k}\left(A_{\Omega}-f^{\prime}(u)\right)$ be the $k^{\text {th }}$ eigenvalue of the operator $A_{\Omega}-f^{\prime}(u)$. Let

$$
S P^{+}(\Omega)=\left\{u \in \operatorname{SP}(\Omega): \lambda_{1}\left(A_{\Omega}-f^{\prime}(u)\right)>0\right\}
$$

be the subset of stable steady points,

$$
S P^{0}(\Omega)=\left\{u \in S P(\Omega): \exists k \in \mathbb{N}, \lambda_{k}\left(A_{\Omega}-f^{\prime}(u)\right)=0\right\}
$$

be the subset of nonhyperbolic steady points, and

$$
S P^{-}(\Omega)=\left\{u \in S P(\Omega): \lambda_{1}\left(A_{\Omega}-f^{\prime}(u)\right)<0 \text { and } \forall k \in \mathbb{N}, \lambda_{k}\left(A_{\Omega}-f^{\prime}(u)\right) \neq 0\right\}
$$

be the subset of unstable steady points. The set $S P^{+}(\Omega) \cup S P^{-}(\Omega)$ is the subset of hyperbolic steady points. It is known (see [H2, Chapter 4]) that each of them is isolated in $L^{2}(\Omega)$. It is also known that stable steady states are attractive in the sense of Lyapunov, and the unstable ones are repulsive for almost all data in their neighborhood.

The condition (7) implies that equilibria and solutions of the parabolic equation with initial conditions in $L^{\infty}(\Omega)$ are uniformly bounded in $L^{\infty}(\Omega)$.

Proposition 2.4 There exists $K>0$ such that

$$
\begin{aligned}
\forall u \in S P(\Omega), & \|u\|_{L^{\infty}(\Omega)} \leqslant K, \\
\forall t>0, & \|u(t)\|_{L^{\infty}(\Omega)} \leqslant \max \left\{K,\left\|u_{0}\right\|_{L^{\infty}(\Omega)}\right\}
\end{aligned}
$$

where $u(t)$ is the solution of (6) with the initial condition $u_{0}$.

Proof. The proof is standard and applies to all weak solutions (see e.g. [GT]). By (7), there exists $K>0$ such that for all $x$ with $|x|>K, f(x) / x<1$. Taking as test functions $(u-k)^{+}$and $(u+k)^{-}$ in (9), we obtain $-K \leqslant u \leqslant K$. The second statement is proved in the same way but using (1) and with $K^{\prime}=\max \left(K,\left\|u_{0}\right\|_{\infty}\right)$. We conclude by the Gronwall lemma.

Let $K$ be the constant given in Proposition 2.4 We denote by $C_{f}^{K}$ the smallest constant such that

$$
|f(x)-f(y)| \leqslant C_{f}^{K}|x-y| \quad \forall x, y \in \bar{B}(0, K) .
$$

Of course $f$ is also bounded on $B(0, K)$.

COROllary 2.5 Under the conditions $f \in C^{1}(\mathbb{R}),(7)$ and $u_{0} \in L^{\infty}(\Omega)$, we may assume that $f$ is globally Lipschitz continuous (and bounded). So, for all $u_{0} \in L^{\infty}(\Omega)$, there exists a unique solution of (6).

See e.g. [H2, Corollary 3.3.5]. This will be crucial in most proofs which follow.

\subsection{Restriction and extension between $D$ and $\Omega \subset D$}

As we will perturb the domain, we need one large space in which solutions can be considered and compared: this is $L^{2}(D), D$ (the design region) being given by $\left(C_{1}\right)$. Let us denote by $\|\cdot\|$ the norm in $\mathcal{L}\left(L^{2}(D)\right)$ and fix the following operators of restriction and extension:

$$
r_{\Omega} \in \mathcal{L}\left(L^{2}(D), L^{2}(\Omega)\right) \quad \text { and } \quad p_{\Omega} \in \mathcal{L}\left(L^{2}(\Omega), L^{2}(D)\right)
$$


defined by

$$
\begin{aligned}
& \forall u \in L^{2}(D), \quad r_{\Omega}(u)=u \text { in } \Omega, \\
& \forall u \in L^{2}(\Omega), \quad p_{\Omega}(u)=u \text { in } \Omega, p_{\Omega}(u)=0 \text { in } \Omega^{c} .
\end{aligned}
$$

When applied to a vector, the operator $p_{\Omega}$ acts on each of its components.

Of course $p_{\Omega}$ is not continuous in $H^{1}(D)$. We note now that the operators $p_{\Omega} \circ\left(\lambda-A_{\Omega}\right)^{-1} \circ r_{\Omega}$ and $p_{\Omega} \circ S_{\Omega}(t) \circ r_{\Omega}$ belong to $\mathcal{L}\left(L^{2}(D)\right)$ and the values of the norms remain unchanged. So, we will consider the resolvent operator $\left(\lambda-A_{\Omega}\right)^{-1}$ as an operator from $L^{2}(D)$ to $L^{2}(D)$. When it does not lead to confusion, we will often omit the operators $p_{\Omega}$ and $r_{\Omega}$. It is clear that the formula (4) remains valid when we consider it in the sense of composition with $p_{\Omega}$ and $r_{\Omega}$.

\subsection{Mosco convergence}

We now introduce the notion of Mosco convergence which appears in our main assumption $\left(C_{4}\right)$. As in other works related to the Neumann perturbation problem [DMa, Dan], we use here actually a special case of the Mosco convergence as introduced in his original paper [Mo, Definition 1.1]. By applying this definition to the linear subspaces of $\left[L^{2}(D)\right]^{N+1}$ defined by

$$
\begin{gathered}
X_{\Omega_{n}}=\left\{\left(p_{\Omega_{n}}(u), p_{\Omega_{n}}(\nabla u)\right): u \in L^{2}\left(\Omega_{n}\right)\right\}, \\
X_{\Omega}=\left\{\left(p_{\Omega}(u), p_{\Omega}(\nabla u)\right): u \in L^{2}(\Omega)\right\},
\end{gathered}
$$

we obtain the following

Definition 2.6 Let $\Omega$ be an open set and $\left(\Omega_{n}\right)_{n \in \mathbb{N}}$ a sequence of open sets. We say that $\left(\Omega_{n}\right)_{n \in \mathbb{N}}$ converges in the sense of Mosco to $\Omega$ if the following conditions $\left(M_{1}\right)$ and $\left(M_{2}\right)$ hold:

$\left(M_{1}\right)$ if $u_{n} \in H^{1}\left(\Omega_{n}\right)$ are such that

$$
p_{\Omega_{n}}\left(u_{n}\right) \stackrel{L^{2}(D)}{\longrightarrow} v, \quad p_{\Omega_{n}}\left(\nabla u_{n}\right) \stackrel{\left[L^{2}(D)\right]^{N}}{\longrightarrow} b,
$$

then there exists $u \in H^{1}(\Omega)$ such that $v=p_{\Omega}(u)$ and $b=p_{\Omega}(\nabla u)$, $\left(M_{2}\right)$ for all $u \in H^{1}(\Omega)$, there exists $u_{n} \in H^{1}\left(\Omega_{n}\right)$ such that

$$
p_{\Omega_{n}}\left(u_{n}\right) \stackrel{L^{2}(D)}{\longrightarrow} p_{\Omega}(u), \quad p_{\Omega_{n}}\left(\nabla u_{n}\right) \stackrel{\left[L^{2}(D)\right]^{N}}{\longrightarrow} p_{\Omega}(\nabla u) .
$$

As noted in the Introduction, it is known that under conditions $\left(M_{1}\right)$ and $\left(M_{2}\right)$, the solution to the linear problem (2) is continuous with respect to domain perturbations; the same was shown for the semilinear case in [DMa]. In Section 4, we will prove this also for solutions of the nonlinear evolution equation (6). In the Introduction we have given more known results about this convergence.

\subsection{Distance between linear spaces}

In order to state some of the convergence results, we want to make precise the notion of distance between sets and between linear spaces. 
Definition 2.7 (see [K, IV.2]) Let $d^{H}$ be the symmetric Hausdorff distance between sets: if $X$ and $Y$ are nonempty subsets of a normed space $(Z,\|\cdot\|)$,

$$
d^{H}(X, Y)=\max \left(\sup _{x \in X} \inf _{y \in Y}\|x-y\|, \sup _{y \in Y} \inf _{x \in X}\|x-y\|\right) .
$$

If $X$ and $Y$ are linear spaces, $d^{H}(X, Y)$ is infinite or 0 . So, let $d$ be the Hausdorff distance between the sets of unit vectors:

$$
d(X, Y)=\max \left(\sup _{\substack{x \in X \\\|x\|=1}} \inf _{\substack{y \in Y \\\|y\|=1}}\|x-y\|, \sup _{\substack{y \in Y \\\|y\|=1}} \inf _{\substack{x \in X \\\|x\|=1}}\|x-y\|\right) .
$$

If we complete this definition by setting

$$
d(X,\{0\})=2 \text { for } X \neq\{0\}, \quad d(\{0\},\{0\})=0,
$$

then $d$ is a distance on the set of all closed subspaces of $Z$.

REMARK 2.8 1) The distance $d$ induces on the set of closed subspaces of $Z$ the same topology as the 'gap' defined as

$$
\delta(X, Y)=\max \left(\sup _{\substack{x \in X \\\|x\|=1}} \inf _{y \in Y}\|x-y\|, \sup _{\substack{y \in Y \\\|y\|=1}} \inf _{x \in X}\|x-y\|\right) .
$$

See [K, IV.2.1]. This 'gap' appears in the statements of many theorems in [K].

2) Note that $d(X, Y)<1$ implies $\operatorname{dim} X=\operatorname{dim} Y$ [K, IV.2.2].

\section{Stability}

In all what follows in this part, we consider a domain $\Omega$ and a sequence of domains $\left(\Omega_{n}\right)_{n \in \mathbb{N}}$ satisfying conditions $\left(C_{1}\right)-\left(C_{4}\right)$. For simplicity, let us write $A_{n}, S_{n}, A, S$ instead of $A_{\Omega_{n}}, S_{\Omega_{n}}, A_{\Omega}$, $S_{\Omega}$ and $p_{n}, p$ instead of $p_{\Omega_{n}}, p_{\Omega}$.

\subsection{Resolvent convergence}

We denote by $R_{A}$ the resolvent operator which to $v$ associates $A^{-1}(f(v))$, extended by zero outside $\Omega$ :

$$
R_{A}: L^{2}(D) \rightarrow L^{2}(D), \quad v \mapsto p\left(A^{-1}(f(v))\right) .
$$

We define $R_{A_{n}}$ in the same way. Note that only $\left.v\right|_{\Omega}$ enters the definition of $R_{A}(v)$, and so we can consider $L^{2}(\Omega)$ as the effective domain of $R_{A}$. We also have

$$
u \in S P(\Omega) \Leftrightarrow R_{A}(u)=u .
$$

It is clear that $R_{A}$ is a compact operator, satisfying, for all $v \in L^{2}(D)$,

$$
\left\|R_{A}(v)\right\|_{H^{1}(\Omega)} \leqslant C_{f}^{K}\|v\|_{L^{2}(D)}
$$

where $C_{f}^{K}$ is defined by $(10)$. The following result will be essential for the proof of Theorem 3.4 
LEMmA 3.1 Suppose that $\left(w_{n}\right)$ is a sequence in $L^{2}(D)$ such that $\left(f\left(w_{n}\right)\right)$ converges weakly to $h$ in $L^{2}(D)$. Then

$$
\lim _{n \rightarrow \infty}\left\|R_{A_{n}}\left(w_{n}\right)-A^{-1}(h)\right\|_{L^{2}(D)}=0, \quad \lim _{n \rightarrow \infty}\left\|R_{A}\left(w_{n}\right)-A^{-1}(h)\right\|_{L^{2}(D)}=0 .
$$

Proof. Let us prove the first claim. Let $u_{n}=R_{A_{n}}\left(w_{n}\right)$. Then

$$
\int_{\Omega_{n}}\left(\nabla u_{n} \nabla \phi_{n}+u_{n} \phi_{n}\right)=\int_{\Omega_{n}} f\left(w_{n}\right) \phi_{n}
$$

for all $\phi_{n} \in H^{1}\left(\Omega_{n}\right)$. It is easy to see that the sequence $\left(p_{n}\left(u_{n}\right), p_{n}\left(\nabla u_{n}\right)\right)$ is bounded in $\left[L^{2}(D)\right]^{N+1}$. So, by $\left(M_{1}\right)$,

$$
\left(p_{n}\left(u_{n}\right), p_{n}\left(\nabla u_{n}\right)\right) \stackrel{\left[L^{2}(D)\right]^{N+1}}{\longrightarrow}(p(u), p(\nabla u)) .
$$

Let $\left(\varphi_{n}\right)$ and $\varphi$ be given by $\left(M_{2}\right)$ and take them as test functions in (13). We can now pass to the limit, obtaining $A u=h$ on $\Omega$. So, $\left(u_{n}\right)$ converges to $A^{-1}(h)$ weakly in $L^{2}(D)$. By $\left(C_{3}\right)$, we find that $\left(u_{n}\right)$ converges to $A^{-1}(h)$ strongly in $L^{2}(\Omega)$. Together with $\left(C_{2}\right)$, this gives

$$
\int_{\Omega_{n}}\left|u_{n}-A^{-1}(h)\right|^{2}=\int_{\Omega}\left|u_{n}-A^{-1}(h)\right|^{2} \rightarrow 0 \quad \text { as } n \rightarrow \infty .
$$

This ends the proof of the first statement. The second one comes from the weak continuity of the operator $A^{-1}$ and the compact injection of $H^{1}(\Omega)$ into $L^{2}(D)$, by $\left(C_{3}\right)$.

REMARK 3.2 Lemma 3.1 remains true for domains which do not satisfy $\left(C_{2}\right)$, but a more general condition $\left|\Omega_{n} \backslash \Omega\right| \rightarrow 0$. One should just replace (14) by

$$
\lim _{n \rightarrow \infty} \int_{D \backslash \Omega}\left|u_{n}-A^{-1}(h)\right|^{2}=0 .
$$

This follows from the boundedness of $f$, which gives the uniform boundedness of $u_{n}=R_{A_{n}}\left(w_{n}\right)$ in $L^{\infty}(D)$.

Lemma 3.3 Let $\left(h_{n}\right)$ be a sequence in $L^{2}(D)$ such that $\left\|h_{n}\right\|_{L^{2}(D)} \leqslant 1$. Let $\lambda>C_{f}^{K}-1$. (We recall that $f$ can be considered Lipschitz continuous with the Lipschitz constant $C_{f}^{K}$.) Then

$$
\lim _{n \rightarrow \infty}\left\|\left(A_{n}+\lambda-f^{\prime}\left(u_{n}\right)\right)^{-1}\left(h_{n}\right)-\left(A+\lambda-f^{\prime}(u)\right)^{-1}\left(h_{n}\right)\right\|_{L^{2}(D)}=0 .
$$

Proof. Up to a subsequence, $h_{n}$ converges weakly in $L^{2}(D)$ to $h$. Let

$$
v_{n}=\left(A_{n}+\lambda-f^{\prime}\left(u_{n}\right)\right)^{-1}\left(h_{n}\right) .
$$

Then

$$
\int_{\Omega_{n}}\left|\nabla v_{n}\right|^{2}+\int_{\Omega_{n}} v_{n}^{2}+\lambda \int_{\Omega_{n}} v_{n}^{2}+\int_{\Omega_{n}} f^{\prime}\left(u_{n}\right) v_{n}^{2}=\int_{\Omega_{n}} h_{n} v_{n},
$$

which gives

$$
\left\|v_{n}\right\|_{H^{1}\left(\Omega_{n}\right)}^{2} \leqslant 1+\frac{1}{1+\lambda-C_{f}^{K}} .
$$


So, by $\left(M_{1}\right)$ there exists $v \in H^{1}(\Omega)$ such that, up to a subsequence,

$$
\left(p_{n}\left(\nabla v_{n}\right), p_{n}\left(v_{n}\right)\right) \stackrel{\left[L^{2}(D)\right]^{N+1}}{\longrightarrow}(p(\nabla v), p(v)) .
$$

Also, by $\left(C_{3}\right), v_{n}$ converges strongly to $v$ in $L^{2}(\Omega)$. Let $\varphi \in H^{1}(\Omega)$ and let $\varphi_{n} \in H^{1}\left(\Omega_{n}\right)$ be given by $\left(M_{2}\right)$. Take them as test functions in the equation defining $v_{n}$ :

$$
\int_{\Omega_{n}}\left\{\nabla v_{n} \nabla \varphi_{n}+(1+\lambda) v_{n} \varphi_{n}-f^{\prime}\left(u_{n}\right) v_{n} \varphi_{n}\right\}=\int_{\Omega_{n}} h_{n} \varphi_{n}
$$

for all $n \in \mathbb{N}$. Passing to the limit, and applying the boundedness of $f^{\prime}$, we obtain $v=(A+\lambda-$ $\left.f^{\prime}(u)\right)^{-1}(h)$. We now use $v_{n}$ as a test function in the above equation. Note that, by $\left(C_{2}\right)$ and $\left(C_{3}\right)$,

$$
\int_{\Omega_{n}} h_{n} v_{n}=\int_{\Omega} h_{n} v_{n} \rightarrow \int_{\Omega} h v
$$

Thus

$$
\lim _{n \rightarrow \infty} \int_{\Omega_{n}}\left\{\left|\nabla v_{n}\right|^{2}+(1+\lambda)\left|v_{n}\right|^{2}-f^{\prime}\left(u_{n}\right)\left|v_{n}\right|^{2}\right\}=\int_{\Omega}\left\{|\nabla v|^{2}+(1+\lambda)|v|^{2}-f^{\prime}(u)|v|^{2}\right\} .
$$

It follows that

$$
\lim _{n \rightarrow \infty} \int_{D}\left\{\left|p_{n}\left(\nabla v_{n}\right)-p(\nabla v)\right|^{2}+\left|p_{n}\left(v_{n}\right)-p(v)\right|^{2}\right\}=0 .
$$

So,

$$
\lim _{n \rightarrow \infty} \int_{D}\left|p_{n}\left(v_{n}\right)-p(v)\right|^{2}=0 .
$$

This means that $\left(A_{n}+\lambda-f^{\prime}\left(u_{n}\right)\right)^{-1}\left(h_{n}\right)$ converges to $\left(A+\lambda-f^{\prime}(u)\right)^{-1}(h)$ in $L^{2}(D)$. On the other hand, it is easy to see that $\left(A+\lambda-f^{\prime}(u)\right)^{-1}\left(h_{n}\right)$ converges to $\left(A+\lambda-f^{\prime}(u)\right)^{-1}(h)$ in $L^{2}(D)$. This ends the proof.

\subsection{Main theorems}

We now establish the continuity of the hyperbolic equilibrium point with respect to our domain perturbation.

THEOREM 3.4 For all $u \in S P^{+}(\Omega) \cup S P^{-}(\Omega)$, there exists $\left(u_{n}\right)_{n \in \mathbb{N}}$ which converges to $u$ in $L^{2}(D)$ and such that $u_{n} \in \operatorname{SP}\left(\Omega_{n}\right)$ for all $n$.

THEOREM 3.5 Let $\left(u_{n}\right)_{n \in \mathbb{N}}$ be a sequence such that $u_{n} \in S P\left(\Omega_{n}\right)$ and which converges to $u$ in $L^{2}(D)$. Then $u \in S P(\Omega)$ and for every $k \in \mathbb{N}$,

$$
\begin{aligned}
\lambda_{k}\left(A_{\Omega_{n}}-f^{\prime}\left(u_{n}\right)\right) & \rightarrow \lambda_{k}\left(A_{\Omega}-f^{\prime}(u)\right) \quad \text { as } n \rightarrow \infty, \\
d\left(W_{n}^{k}, W^{k}\right) & \rightarrow 0 \quad \text { as } n \rightarrow \infty .
\end{aligned}
$$

Here $d$ is the distance between closed subspaces of $L^{2}(D)$, as in Definition 2.7 and $W^{k}, W_{n}^{k}$ are the subspaces generated by the first $k$ eigenvectors:

$$
W^{k}=\operatorname{span}\left[e^{1} ; \ldots ; e^{k}\right], \quad W_{n}^{k}=\operatorname{span}\left[e_{n}^{1} ; \ldots ; e_{n}^{k}\right]
$$

where $e^{k}$ is the eigenvector corresponding to the eigenvalue $\lambda_{k}\left(A_{\Omega}-f^{\prime}(u)\right)$, and $e_{n}^{k}$ the eigenvector corresponding to the eigenvalue $\lambda_{k}\left(A_{\Omega_{n}}-f^{\prime}\left(u_{n}\right)\right)$. 
Without loss of generality, by Proposition 2.4 and Corollary 2.5, we can suppose that $f$ is Lipschitz continuous with Lip $f=C_{f}^{K}$. Here again, we assume that the functions are extended by zero outside the open set in which they are naturally defined. To prove this result, we will use the Leray-Schauder fixed-point index (see e.g. [Z, Vol. I, Chapter 12]).

Proof of Theorem 3.4 Let $u \in S P^{+}(\Omega) \cup S P^{-}(\Omega)$. Being hyperbolic, $u$ is isolated, i.e. for $\varepsilon$ small enough, $u$ is the unique fixed point of $R_{A}$ in $\bar{B}(u, \varepsilon)$, where $B(u, \varepsilon)$ denotes the ball in $L^{2}(D)$ of center $u$ and radius $\varepsilon$. Let $i(\cdot, \cdot)$ be the Leray-Schauder fixed-point index. We then have $i\left(R_{A}, B(u, \varepsilon)\right) \neq 0$. Let $H_{n}: \bar{B}(u, \varepsilon) \times[0,1] \rightarrow L^{2}(D)$ be defined by

$$
H_{n}(x, t)=t R_{A_{n}}(x)+(1-t) R_{A}(x) .
$$

Suppose that, for $n$ large enough, $H_{n}$ is a compact homotopy. Then

$$
i\left(R_{A_{n}}, B(u, \varepsilon)\right)=i\left(R_{A}, B(u, \varepsilon)\right) \neq 0 .
$$

This implies that there exists $u_{n} \in S P\left(\Omega_{n}\right) \cap B(u, \varepsilon)$. By a direct application of Lemma 3.1 we find that the sequence $\left(u_{n}\right)$ converges strongly to $u$ in $L^{2}(D)$ : this would end the proof.

In order to prove that $H_{n}$ is a compact homotopy, we have to verify that $H_{n}$ is compact and that $H_{n}(x, t) \neq x$ for all $(x, t) \in \partial B(u, \varepsilon) \times[0,1]$. The compactness of $H_{n}$ follows from the compactness of $A_{n}^{-1}$ and $A^{-1}$. Suppose that there exists a sequence $\left(v_{k}, t_{k}\right) \in \partial B(u, \varepsilon) \times[0,1]$ such that $H_{n}\left(v_{k}, t_{k}\right)=v_{k}$. Note that for a subsequence, after renumbering, we can assume that $H_{n}\left(v_{n}, t_{n}\right)=v_{n}$. Let $v$ be the weak limit in $L^{2}(D)$ of $v_{n}$, and $h$ the weak limit of $f\left(v_{n}\right)$. Since $H_{n}\left(v_{n}, t_{n}\right)=v_{n}$, the sequence $\left(v_{n}\right)$ converges strongly to $v \in \partial B(u, \varepsilon)$ in $L^{2}(D)$ and as $f$ is Lipschitz continuous, $h=f(v)$. On the other hand, by Lemma 3.1. $\left(R_{A_{n}}\left(v_{n}\right)\right)_{n}$ and $\left(R_{A}\left(v_{n}\right)\right)_{n}$ converge strongly in $L^{2}(D)$ to $A^{-1}(h)=A^{-1}(f(v))$. So, by $15,, v=R_{A}(v)$. This contradicts the fact that $u$ is the unique fixed point in $\bar{B}(u, \varepsilon)$.

Proof of Theorem 3.5 The fact that $u \in S P(\Omega)$ is clear. The two convergences come (see [K] IV, 3.4-3.5]) from Lemma 3.3 the convergence of the resolvent operators $\left(A_{n}+\lambda-f^{\prime}\left(u_{n}\right)\right)^{-1}$ to the resolvent operator $\left(A+\lambda-f^{\prime}(u)\right)^{-1}$ in $\mathcal{L}\left(L^{2}(D)\right)$. Note that these operators have of course the same eigenvalues and eigenspaces as $\left(A_{n}-f^{\prime}\left(u_{n}\right)\right)^{-1},\left(A-f^{\prime}(u)\right)^{-1}$.

REMARK 3.6 1) We do not need $\left(C_{2}\right)$ for Theorem 3.4 , but it is crucial for the proof of Theorem 3.5. However, $\left(C_{2}\right)$ is of course not a necessary condition for having Theorem 3.5

We will show now that if the flux in the domain $\Omega$ has no nonhyperbolic equilibrium, then the number of equilibria is the same in the limit and in the perturbed domains. We begin with a lemma referring to a slightly more general situation.

LEMma 3.7 Let $u \in S P^{+}(\Omega) \cup S P^{-}(\Omega)$. If there exist two sequences $\left(u_{n}\right),\left(v_{n}\right)$ with $u_{n} \in S P\left(\Omega_{n}\right)$ and $v_{n} \in S P\left(\Omega_{n}\right)$ both converging to $u$ in $L^{2}(D)$, then, for $n$ large enough, $u_{n}=v_{n}$.

Proof. Assume that $u_{n} \neq v_{n}$ on a set of positive measure. Define $w_{n}=\left(u_{n}-v_{n}\right) /\left\|u_{n}-v_{n}\right\|_{L^{2}\left(\Omega_{n}\right)}$. Note that by $\left(C_{2}\right),\left\|w_{n}\right\|_{L^{2}(\Omega)}=\left\|w_{n}\right\|_{L^{2}\left(\Omega_{n}\right)}=1$. We will show that

$$
w_{n} \rightarrow 0 \quad \text { strongly in } L^{2}(\Omega),
$$

which will obviously be a contradiction. 
From the equation solved by $u_{n}-v_{n}$ we see that

$$
\left\|u_{n}-v_{n}\right\|_{H^{1}\left(\Omega_{n}\right)} \leqslant C_{f}\left\|u_{n}-v_{n}\right\|_{L^{2}\left(\Omega_{n}\right)} .
$$

So, $\left\|u_{n}-v_{n}\right\|_{H^{1}\left(\Omega_{n}\right)} \rightarrow 0$. Note also that $w_{n} \in H^{1}\left(\Omega_{n}\right)$ and is a solution of

$$
\int_{\Omega_{n}} \nabla w_{n} \nabla \varphi_{n}+\int_{\Omega_{n}} w_{n} \varphi_{n}=\int_{\Omega_{n}} \frac{f\left(u_{n}\right)-f\left(v_{n}\right)}{\left\|u_{n}-v_{n}\right\|_{L^{2}\left(\Omega_{n}\right)}} \varphi_{n}, \quad \forall \varphi_{n} \in H^{1}\left(\Omega_{n}\right),
$$

which by $\left(C_{2}\right)$ is the same as

$$
\int_{\Omega} \nabla w_{n} \nabla \varphi_{n}+\int_{\Omega} w_{n} \varphi_{n}=\int_{\Omega} \frac{f\left(u_{n}\right)-f\left(v_{n}\right)}{\left\|u_{n}-v_{n}\right\|_{L^{2}(\Omega)}} \varphi_{n}, \quad \forall \varphi_{n} \in H^{1}\left(\Omega_{n}\right) .
$$

Taking $w_{n}$ as a test function in (17) implies that $\left\|w_{n}\right\|_{H^{1}\left(\Omega_{n}\right)}$ is uniformly bounded. So, up to a subsequence, thanks to the Mosco condition $\left(M_{1}\right)$, there exists $w \in H^{1}(\Omega)$ such that

$$
\left(p_{n}\left(\nabla w_{n}\right), p_{n}\left(w_{n}\right)\right) \stackrel{\left[L^{2}(D)\right]^{N+1}}{\longrightarrow}(p(\nabla w), p(w)) .
$$

We will now prove that $w$ is a solution of

$$
\int_{\Omega} \nabla w \nabla \varphi+\int_{\Omega} w \varphi=\int_{\Omega} f^{\prime}(u) w \varphi, \quad \forall \varphi \in H^{1}(\Omega) .
$$

This, since $u \in S P^{+}(\Omega) \cup S P^{-}(\Omega)$, gives us $w=0$. Let $\varphi \in H^{1}(\Omega)$ and let $\varphi_{n} \in H^{1}\left(\Omega_{n}\right)$ be the sequence given by the Mosco condition $\left(M_{2}\right)$. Passing to the limit in (17), in order to obtain (19), we have to prove that

$$
\lim _{n \rightarrow \infty} \int_{\Omega} \frac{f\left(u_{n}\right)-f\left(v_{n}\right)}{\left\|u_{n}-v_{n}\right\|_{L^{2}\left(\Omega_{n}\right)}} \varphi_{n}=\int_{\Omega} f^{\prime}(u) w \varphi .
$$

Since the injection from $H^{1}(\Omega)$ into $L^{2}(\Omega)$ is compact by $\left(C_{3}\right)$, the function $f$ can be considered to belong to $\mathcal{L}\left(H^{1}(\Omega), L^{2}(\Omega)\right)$, and

$$
\left\|\frac{f\left(v_{n}\right)-f\left(u_{n}\right)}{\left\|u_{n}-v_{n}\right\|_{L^{2}\left(\Omega_{n}\right)}}-f^{\prime}\left(u_{n}\right) w_{n}\right\|_{L^{2}(\Omega)}=\frac{o\left(\left\|u_{n}-v_{n}\right\|_{H^{1}(\Omega)}\right)}{\left\|u_{n}-v_{n}\right\|_{L^{2}\left(\Omega_{n}\right)}} .
$$

By (16) we have

$$
\frac{o\left(\left\|u_{n}-v_{n}\right\|_{H^{1}(\Omega)}\right.}{\left.\left\|u_{n}-v_{n}\right\|_{L^{2}\left(\Omega_{n}\right)}\right)} \leqslant C_{f} \frac{o\left(\left\|u_{n}-v_{n}\right\|_{H^{1}(\Omega)}\right)}{\left\|u_{n}-v_{n}\right\|_{H^{1}(\Omega)}} .
$$

Since $\left\|u_{n}-v_{n}\right\|_{H^{1}(\Omega)} \rightarrow 0$, it follows that

$$
\frac{f\left(v_{n}\right)-f\left(u_{n}\right)}{\left\|u_{n}-v_{n}\right\|_{L^{2}\left(\Omega_{n}\right)}}-f^{\prime}\left(u_{n}\right) w_{n} \rightarrow 0 \quad \text { in } L^{2}(\Omega) .
$$

Thus, we can pass to the limit in (17), obtain (19), and conclude that $w=0$. We got our contradiction: (a subsequence of) $w_{n}$ converges strongly in $L^{2}(\Omega)$ to 0 and $\left\|w_{n}\right\|_{L^{2}(\Omega)}=1$. 
Thus, if no nonhyperbolic equilibria exist, the structure of the set of equilibria in $\Omega_{n}$ is very similar to the one in the limit domain.

THEOREM 3.8 Suppose that $S P(\Omega)^{0}=\emptyset$. Then for $n$ large enough,

$$
\operatorname{card} S P^{+}\left(\Omega_{n}\right)=\operatorname{card} S P^{+}(\Omega), \quad \operatorname{card} S P^{-}\left(\Omega_{n}\right)=\operatorname{card} S P^{-}(\Omega) .
$$

Proof. This is an immediate consequence of Theorems 3.4 and 3.5 and of Lemma 3.7

THEOREM 3.9 Suppose that $\operatorname{SP}(\Omega)^{0}=\emptyset$. Then

$$
\lim _{n \rightarrow \infty} d^{H}\left(S P^{+}\left(\Omega_{n}\right), S P^{+}(\Omega)\right)=0, \quad \lim _{n \rightarrow \infty} d^{H}\left(S P^{-}\left(\Omega_{n}\right), S P^{-}(\Omega)\right)=0 .
$$

Here, $d^{H}$ denotes the Hausdorff distance between sets in $L^{2}(D)$. The functions are considered, as usual, in $L^{2}(D)$ by extension by zero.

Proof. The assumption 7 on $f$ implies that $S P(\Omega)$ is bounded in $H^{1}(\Omega)$ and hence compact in $L^{2}(\Omega)$. So, if $S P(\Omega)$ is hyperbolic, the subset $S P^{+}(\Omega) \cup S P^{-}(\Omega)$ is finite. It is then clear, by Theorems 3.4 and 3.5 , that

$$
\lim _{n \rightarrow \infty} \sup _{u \in S P^{+}(\Omega)} \inf _{v \in S P^{+}\left(\Omega_{n}\right)}\|u-v\|_{L^{2}(D)}=0
$$

On the other hand, let $u_{n} \in S P^{+}\left(\Omega_{n}\right)$. As $S P\left(\Omega_{n}\right)$ is uniformly bounded with respect to $n$ in $H^{1}\left(\Omega_{n}\right)$, using the Mosco conditions, it is easy to see that, up to a subsequence, $u_{n}$ converge strongly to $u \in S P(\Omega)$ in $L^{2}(\Omega)$. Since $u_{n} \in L^{\infty}\left(\Omega_{n}\right)$, the convergence holds in $L^{2}(D)$. Using Theorem 3.5 again, we conclude that $u \in S P^{+}(\Omega)$ and so

$$
\lim _{n \rightarrow \infty} \sup _{v \in S P^{+}\left(\Omega_{n}\right)} \inf _{u \in S P^{+}(\Omega)}\|u-v\|_{L^{2}(D)}=0 .
$$

This ends the proof of the first claim. For the second, we use the same argument. Moreover, from the first Mosco condition, we have $\lim \sup \lambda\left(\Omega_{n}\right) \leqslant \lambda(\Omega)$ so if $\left(u_{n}\right)$ is a sequence in $S P^{-}\left(\Omega_{n}\right)$, then each limit of a subsequence is in $S P^{-}(\Omega) \cup S P^{0}(\Omega)$, so in $S P^{-}(\Omega)$. This simplifies the proof in this case.

\section{Convergence of semigroups}

We are now interested in the continuity of parabolic Neumann problems under the perturbation of domain defined above. According to Corollary 2.5. if we restrict the space of initial conditions to $L^{\infty}(D)$, we can suppose that $f$ is globally Lipschitz continuous.

We know that under the condition $(G)$ or $(7)$, for all $u_{0} \in L^{2}(\Omega)$, there exists a unique solution of $(6)$. The map $T_{\Omega}: \mathbb{R}^{+} \times L^{2}(\Omega) \rightarrow L^{2}(\Omega)$ which to $\left(t, u_{0}\right)$ associates the solution of the equation (6) is a nonlinear semigroup. Let us make this more specific. Take $\beta>0$ and $T>0$. We define $L_{\beta}^{2}\left((0, T), L^{2}(D)\right)$ to be the Banach space of functions defined on $(0, T) \times \Omega$, endowed with the norm

$$
\left(\int_{0}^{T} e^{-\beta t}\|u(t)\|_{L^{2}(D)}^{2} \mathrm{~d} t\right)^{1 / 2} .
$$


Let $\mathcal{F}: L_{\beta}^{2}\left((0, T), L^{2}(D)\right) \rightarrow L_{\beta}^{2}\left((0, T), L^{2}(D)\right)$ be the map

$$
\mathcal{F}(u)(t)=\int_{0}^{t} S(t-s) f(u(s)) \mathrm{d} s .
$$

We deduce from $(5)$ by a simple calculation that $\mathcal{F}$ is Lipschitz with a constant smaller than Lip $f / \sqrt{\beta+2}$. Hence, for $\beta$ large enough, it is strictly contractive, uniformly in $T$ and in $\Omega$.

The solution of 88 is then the unique fixed point in $L_{\beta}^{2}\left((0, T), L^{2}(D)\right)$ of the map $\mathcal{G}$ : $L_{\beta}^{2}\left((0, T), L^{2}(D)\right) \rightarrow L_{\beta}^{2}\left((0, T), L^{2}(D)\right)$ given by

$$
\mathcal{G}(u)(t)=S(t) u_{0}+\mathcal{F}(u)(t) .
$$

We define in the same way the maps $\mathcal{F}_{n}$ and $\mathcal{G}_{n}$ for the semigroup $S_{n}$; they are uniformly contractive as well, with respect to $T$ and $n$.

THEOREM 4.1 Suppose that $\left(\Omega_{n}\right)_{n \in \mathbb{N}}$ converges to $\Omega$ in the sense of Mosco. For all $u_{0} \in L^{\infty}(D)$ and all $T>0$,

$$
\lim _{n \rightarrow \infty}\left\|T_{n}(t) u_{0}-T(t) u_{0}\right\|_{L_{\beta}^{2}\left((0, T), L^{2}(D)\right)}=0
$$

where $\beta$ is such that $\sqrt{C_{f} /(\beta+1)}<1$.

In order to prove this result, we need the resolvent operator and the linear semigroup continuity.

LEMmA 4.2 Assume that $\left(\Omega_{n}\right)$ converges to $\Omega$ in the sense of Mosco. For all $h \in L^{2}(D)$, and all $\lambda \in \mathcal{S}$,

$$
\lim _{n \rightarrow \infty}\left\|\left(\lambda-A_{n}\right)^{-1}(h)-(\lambda-A)^{-1}(h)\right\|_{L^{2}(D)}=0 .
$$

Proof. We will argue as in the proofs of Lemma 3.1 and Theorem 3.5. Let $u_{n}=\left(\lambda-A_{n}\right)^{-1}(h)$. By the inequality 3 and $\left(M_{1}\right)$ there exists $u \in H^{1}(\Omega)$ such that, up to a subsequence,

$$
\left(p_{n}\left(\nabla u_{n}\right), p_{n}\left(u_{n}\right)\right) \stackrel{\left[L^{2}(D)\right]^{N+1}}{\longrightarrow}(p(\nabla u), p(u)) .
$$

Let $\varphi \in H^{1}(\Omega)$ and let $\varphi_{n} \in H^{1}\left(\Omega_{n}\right)$ be given by $\left(M_{2}\right)$. Take them as test functions in the eigenvalue equation for $u_{n}$ :

$$
\int_{\Omega_{n}}\left\{\nabla u_{n} \nabla \varphi_{n}+u_{n} \varphi_{n}-\lambda u_{n} \varphi_{n}\right\}=\int_{\Omega_{n}} h \varphi_{n}
$$

for all $n \in \mathbb{N}$. Passing to the limit, we obtain $u=(\lambda-A)^{-1}(h)$. Using $u_{n}$ as a test function in the above equation, as $\int_{\Omega_{n}} h u_{n} \longrightarrow \int_{\Omega} h u$, we obtain

$$
\lim _{n \rightarrow \infty} \int_{\Omega_{n}}\left\{\left|\nabla u_{n}\right|^{2}+\left|u_{n}\right|^{2}-\lambda\left|u_{n}\right|^{2}\right\}=\int_{\Omega}\left\{|\nabla u|^{2}+|u|^{2}-\lambda|u|^{2}\right\} .
$$

It follows that

$$
\lim _{n \rightarrow \infty} \int_{D}\left\{\left|p_{n}\left(\nabla u_{n}\right)-p(\nabla u)\right|^{2}+(1-\lambda)\left|p_{n}\left(u_{n}\right)-p(u)\right|^{2}\right\}=0 .
$$


Since $\lambda \in \mathcal{S}$, if its imaginary part is equal to zero, then $\lambda<1$. We get

$$
\lim _{n \rightarrow \infty} \int_{D}\left|p_{n}\left(u_{n}\right)-p(u)\right|^{2}=0,
$$

which is the desired result.

COROLlaRY 4.3 For all compact $K \subset \mathcal{S}$ and $h \in L^{2}(D)$,

$$
\lim _{n \rightarrow \infty} \sup _{\lambda \in K}\left\|\left(\lambda-A_{n}\right)^{-1}(h)-(\lambda-A)^{-1}(h)\right\|=0 .
$$

Proof. This is a direct consequence of the resolvent identity. Let $r_{0}=\min \{|\lambda-1|: \lambda \in K\}$. Since $1 \notin \mathcal{S}$, we have $r_{0}>0$. Let $\left(\lambda_{n}\right)_{n \in \mathbb{N}}$ be a maximizing sequence. Then, up to a subsequence, it converges to some $\lambda$. On the other hand, by the resolvent identity, we have

$$
\begin{aligned}
& \left\|\left(\lambda_{n}-A_{n}\right)^{-1} h-\left(\lambda_{n}-A\right)^{-1} h\right\|_{L^{2}(D)} \\
& \qquad \frac{8\left|\lambda_{n}-\lambda\right|}{r_{0}^{2}}\|h\|+\left\|\left(\lambda-A_{n}\right)^{-1} h-(\lambda-A)^{-1} h\right\|_{L^{2}(D)} .
\end{aligned}
$$

We now apply Lemma 4.2 and the proof is finished.

As a consequence of Lemma 4.2 , we prove the continuity of the linear semigroup.

LEMma 4.4 Suppose that $\left(\Omega_{n}\right)_{n \in \mathbb{N}}$ converges to $\Omega$ in the sense of Mosco. For all $u_{0} \in L^{2}(D)$ and all $T, \delta$ such that $0<\delta<T$,

$$
\lim _{n \rightarrow \infty} \sup _{[\delta, T]}\left\|S_{n}(t) u_{0}-S(t) u_{0}\right\|_{L^{2}(D)}=0 .
$$

Proof. Let $\Gamma$ be a smooth contour included in $-\mathcal{S}$, i.e. in the region $\{\lambda: 0 \leqslant|\arg (\lambda+1)| \leqslant$ $2 \pi / 3, \lambda \neq-1\}$, and such that for some $R_{0}>0$,

$$
\lambda \in \Gamma \backslash B\left(-1, R_{0}\right) \Rightarrow \lambda=-1+r e^{ \pm i \theta},
$$

with some fixed $\theta \in(\pi / 2,2 \pi / 3)$ and $r \in\left(R_{0}, \infty\right)$. Using (4), we have, for all $t>0$,

$$
S_{n}(t) u_{0}-S(t) u_{0}=\frac{1}{2 i \pi} \int_{\Gamma} e^{\lambda t}\left[\left(\lambda+A_{n}\right)^{-1} u_{0}-(\lambda+A)^{-1} u_{0}\right] \mathrm{d} \lambda .
$$

Using (3), for all $R>R_{0}$ we obtain the following inequalities, where $r=|\lambda+1|$ :

$$
\begin{aligned}
\| \frac{1}{2 i \pi} \int_{\Gamma \cap \bar{B}(1, R)^{c}} e^{\lambda t}[(\lambda & \left.\left.+A_{n}\right)^{-1} u_{0}-(\lambda+A)^{-1} u_{0}\right] \mathrm{d} \lambda \|_{L^{2}(D)} \\
& \leqslant \frac{2}{\pi}\left\|u_{0}\right\|_{L^{2}(D)} \int_{\Gamma \cap \bar{B}(1, R)^{c}} \frac{\left|e^{\lambda t}\right|}{r} d|\lambda| \\
& \leqslant \frac{4}{\pi}\left\|u_{0}\right\|_{L^{2}(D)} \int_{R}^{\infty} \frac{e^{-t+r t \cos \theta}}{R} d r \leqslant-\frac{4}{\pi}\left\|u_{0}\right\|_{L^{2}(D)} \frac{e^{-t+R t \cos \theta}}{R t \cos \theta},
\end{aligned}
$$


since $\pi>\theta>\pi / 2$. Also, for these values of $\theta$,

$$
\lim _{R \rightarrow \infty} \sup _{[\delta, T]} \frac{e^{t(-1+R \cos \theta)}}{R t \cos \theta}=0 .
$$

Fix now $\varepsilon>0$. There exists $R>0$ such that

$$
\begin{aligned}
\sup _{t \in[\delta, T]} \| S_{n}(t) u_{0}-S(t) & u_{0} \|_{L^{2}(D)} \\
& \leqslant \frac{1}{2 \pi} \int_{\Gamma \cap \bar{B}(1, R)}\left|e^{\lambda T}\right|\left\|\left(\lambda+A_{n}\right)^{-1} u_{0}-(\lambda+A)^{-1} u_{0}\right\|_{L^{2}(D)} d \lambda+\varepsilon
\end{aligned}
$$

The result follows by Corollary 4.3

We are in position to prove Theorem 4.1

Proof of Theorem 4.1 Since $\mathcal{G}_{n}$ are uniformly contractive in $L_{\beta}^{2}\left((0, T), L^{2}(D)\right)$, it is sufficient to prove that for all $p \in \mathbb{N}$ and $\varphi \in L_{\beta}^{2}\left((0, T), L^{2}(D)\right), \mathcal{G}_{n}^{p}(\varphi)$ converges to $\mathcal{G}_{n}^{p}(\varphi)$ strongly in $L_{\beta}^{2}\left((0, T), L^{2}(D)\right)$. Proceeding by recurrence, we have to prove that

$$
\text { if } \quad \varphi_{n} \rightarrow \varphi \text { in } L_{\beta}^{2}\left((0, T), L^{2}(D)\right), \quad \text { then } \quad \mathcal{G}_{n}\left(\varphi_{n}\right) \rightarrow \mathcal{G}_{n}(\varphi) \text { in } L_{\beta}^{2}\left((0, T), L^{2}(D)\right) .
$$

It is clear, by Lemma 4.4 that $S_{n}(t) u_{0} \rightarrow S(t) u_{0}$ in $L_{\beta}^{2}\left((0, T), L^{2}(D)\right)$. Hence it is sufficient to prove that $\mathcal{F}_{n}\left(\varphi_{n}\right) \rightarrow \mathcal{F}_{n}(\varphi)$ in $L_{\beta}^{2}\left((0, T), L^{2}(D)\right)$. Since $\mathcal{F}_{n}$ are uniformly Lipschitz continuous, we have to prove that for all $\varphi \in L_{\beta}^{2}\left((0, T), L^{2}(D)\right)$,

$$
\mathcal{F}_{n}\left(\varphi_{n}\right) \rightarrow \mathcal{F}_{n}(\varphi) \quad \text { in } L_{\beta}^{2}\left((0, T), L^{2}(D)\right) .
$$

Lemma 4.4 implies that $S_{n}(t-s) f(\varphi(s)) \rightarrow S(t-s) f(\varphi(s))$ in $L^{2}(D)$ a.e. in $(0, t)$. Since

$$
\left\|S_{n}(t-s) f(\varphi(s))\right\|_{L^{2}(D)} \leqslant e^{-(t-s)}\left(C+C_{f}^{K}\|\varphi(s)\|_{L^{2}(D)}\right)
$$

with $\varphi(s) \in L_{\beta}^{2}\left((0, T), L^{2}(D)\right)$, the result follows by the Dominated Convergence Theorem.

\section{Appendix}

We present here more details on the numerical simulations, results of which were shown in the Introduction. Recall that we have taken the Allen-Cahn nonlinearity

$$
g(u)=u\left(1-u^{2}\right),
$$

having two stable zeros 1 and -1 , and one unstable equal to 0 . We apply to our equation (1) the following semi-implicit scheme based on the concave-convex splitting of the free energy introduced by Eyre $[\mathrm{E}]$ and explored in view of unconditional stability for the Allen-Cahn and Cahn-Hilliard equations by Vollmayr-Lee and Rutenberg in [VR]:

$$
\frac{u-\tilde{u}}{\tau}-\Delta u=(1-a) u+a \tilde{u}-\tilde{u}^{3},
$$



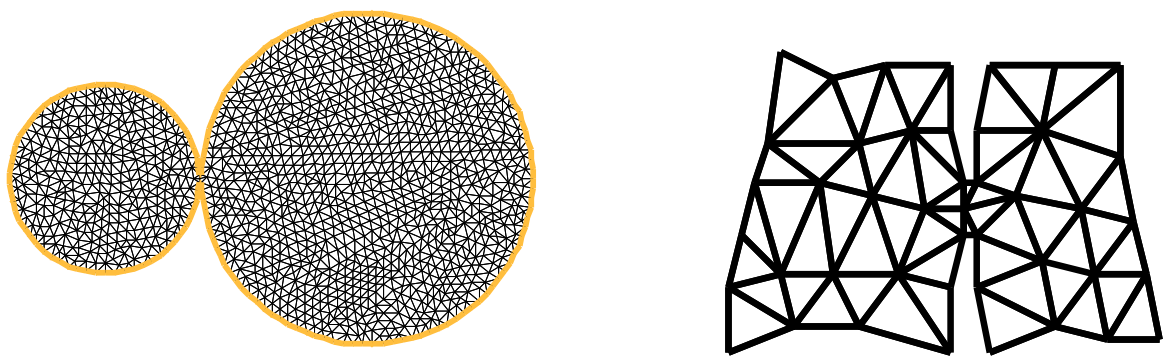

FIG. 4. The domain with its FEM grid and a close-up of the grid near the junction of the two subdomains.

where $\tilde{u}$ is the value from the previous time step. In the experiments we have taken $a=3$, a value for which the time-discrete scheme is stable [VR]. We then use the finite element approximation for the space discretization.

The geometry of the domain and the triangulation are shown in Figure 4. This geometry does not follow precisely our model of Figure 1 , but is very close to it. Figure 4 presents a close-up of the junction of the two subdomains. The domain's dimension are:

$$
\begin{array}{ll}
r_{1}=\frac{1}{3} * 20 & \text { radius of the left ball, } \\
r_{2}=\frac{1}{3} * 35 & \text { radius of the right ball, } \\
r_{0}=\frac{1}{3} & \text { dimension of the 'hole' }
\end{array}
$$

and the numerical parameters are:

$$
\begin{aligned}
\tau & =0.001 & & \text { time-step size }, \\
h_{\max } & =\frac{1}{3} * 1.75 & & \text { diameter of the triangulation, } \\
N & =5366 & & \text { number of nodes in the triangulation. }
\end{aligned}
$$

The scaling factor $\kappa=10 / 3$ is actually included in the equation: we multiply $\Delta u$ by $1 / \kappa^{2}$ and work with the equation $u_{t}-\left(1 / \kappa^{2}\right) \Delta u=g(u)$ and smaller geometrical dimensions.

Results of three simulations are shown in Figure 2

For an insight into the dynamics of the process under study, let us plot a measure of the rate of change of the function $u$ in time, defined as

$$
m\left(t_{n}\right)=\int_{\Omega} \frac{\left|u^{n}-u^{n-1}\right|}{\tau} .
$$

Here $n$ is the time step, and $u^{n}$ the numerical solution at time step $n$, i.e. at $t_{n}=n \tau$. The measure is more sensitive to changes of $u$ than the rate of change of mass (where the mass is defined to be $\int_{\Omega} u$ ), in particular, $m\left(t_{n}\right)=0$ implies $u^{n} \equiv u^{n-1}$ a.e. Figure 5 presents the measure for each of the experiments. Note that a logarithmic scale is used for the vertical axes.

One can see that the evolution speed is (i) nearly constant but increasing on long intervals of time, and (ii) changing rapidly on some very short intervals. This phenomenon is known to 


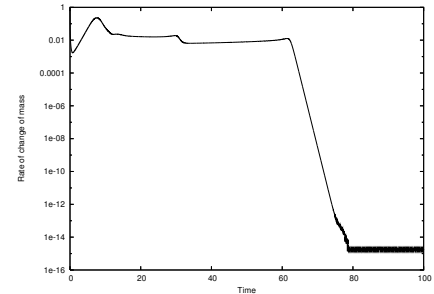

(a)

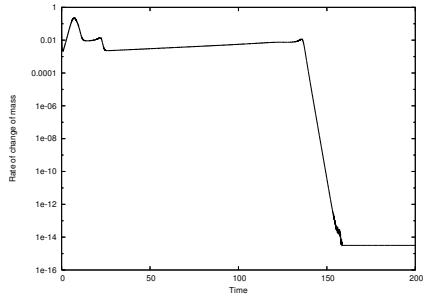

(b)

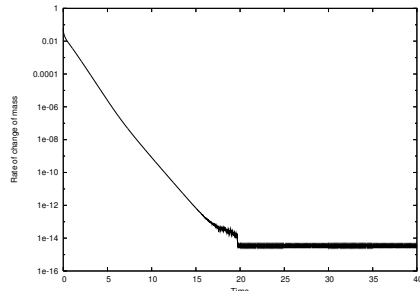

(c)

FIG. 5. The rate of mass change 25, for experiments of Figure 2

correspond to the evolution on the attractor, (i) following the invariant manifolds and (ii) near the unstable equilibria (see for instance $[\mathrm{FH}]$ for the Allen-Cahn case). Our graphs also show a much slower and flatter part by the end of all the experiments, which makes one think there is a particular feature of the final state-this is its stability. This is particularly meaningful when we compare the graph (a) with (b), (c), as the stability of the final state of (a) is clear.

\section{REFERENCES}

[AC] ARrieta, J. M., \& CARVAlho, A. N. Spectral convergence and nonlinear dynamics of reactiondiffusion equations under perturbations of the domain. J. Differential Equations 199 (2004), 143-178. Zbl 1058.35028 MR 2041515

[ACl] Arrieta, J. M., Carvalho, A. N., \& Lozada-Cruz, G. Dynamics in dumbbell domains I. Continuity of the set of equilibria. J. Differential Equations 231 (2006), 551-597. Zbl 1110.35028 MR 2287897

[BV] Bucur, D., \& VARChOn, N. Boundary variation for a Neumann problem. Ann. Scuola Norm. Sup. Pisa Cl. Sci. (4) 24 (2000), 807-821. MR 1822408

[CH] Casten, R. G., \& Holland, C. J. Instability results for reaction-diffusion equations with Neumann boundary conditions. J. Differential Equations 27 (1978), 266-273. Zbl 0338.35055 MR 0480282

[Cor] Corteshani, G. Asymptotic behavior of a sequence of Neumann problems. Comm. Partial Differential Equations 22 (1997), 1691-1729. ZZbl 0895.35011| MR 1469587|

[DMa] Dal Maso, G., Ebobisse, F., \& Ponsiglione, M. A stability result for nonlinear Neumann problems under boundary variations. J. Math. Pures Appl. 82 (2003), 503-532. Zbl 1030.35056 MR 1995490

[Dam] Damlamian, A. Le problème de la passoire de Neumann. Rend. Sem. Mat. Torino 43 (1985), 427-450. Zbl 0629.35031 MR 0884870

[Dan] DANERs, D. Perturbation of semi-linear evolution equations under weak assumptions at initial time. J. Differential Equations 210 (2005), 352-382.

[EG] Evans, L. C., \& GARIEPY, R. F. Measure Theory and Fine Properties of Functions. CRC Press, Boca Raton, FL (1992). Zbl 0804.28001 MR 1158660

[E] EYRE, D. J. An unconditionally stable one-step scheme for gradient systems. Preprint (1997), Univ. of Utah, Salt Lake City.

[FH] Fusco, G., \& HALE, J. K. Slow-motion manifolds, dormant instability, and singular perturbations. \begin{tabular}{lll|l|}
\hline J. Dynam. Differential Equations 1 (1989), 75-94. Zbl 0684.34055 & MR 1010961 \\
\hline
\end{tabular} 
[GT] Gilbarg, D., \& Trudinger, N. S. Elliptic Partial Differential Equations of Second Order. Springer (1977). Zbl 0361.35003 MR 0473443

[GV] GOKIELI, M., \& VARCHON, N. The reaction-diffusion problem on dumbbell domains. Gakuto Int. Ser. Math. Sci. Appl. 2 (2005), 55-72. Zbl 1173.37060 MR 2232109

[HV] Hale, J. K., \& Vegas, J. A nonlinear parabolic equation with varying domain. Arch. Ration. Mech. Anal. 86 (1984), 99-123. Zbl 0569.35048 MR 0751304

[H1] Henrot, A. Continuity with respect to the domain for the Laplacian: A survey. Control Cybernet. 23 (1994), 427-443. Zbl 0822.35029 MR 1303362

[H2] Henry, D. Geometric Theory of Semilinear Parabolic Equations. Lecture Notes in Math. 840, Springer (1981). Zbl 0456.35001 MR 0610244

[J1] JIMво, S. The singularly perturbed domain and the characterization for the eigenfunctions with Neumann boundary condition. J. Differential Equations 77 (1989), 322-350. Zbl 0703.35138 MR 0983298

[J2] Jimbо, S. Singular perturbation of domains and semilinear elliptic equations. III. Hokkaido Math. J. 33 (2004), 11-45. Zbl 1152.35310 MR 2034806

[JM] Jimbo, S., \& MoRITA, Y. Remarks on the behavior of certain eigenvalues on a singularly perturbed domain with several thin channels. Comm. Partial Differential Equations 17 (1992), $523-$ 552. Zbl 0766.35029 MR 1163435

[K] Kato, T. Perturbation Theory for Linear Operators. Springer (1966). Zbl 0148.12601 MR 0203473

[M] Matano, H. Asymptotic behavior and stability of solutions of semilinear diffusion equations. Publ. Res. Inst. Math. Sci. 15 (1979), 401-454. Zbl 0445.35063 MR 0555661

[Maz] MaZ'YA, V. G., \& Poborchi, S. V. Differentiable Functions on Bad Domains. World Sci. (1997). Zbl 0918.46033 MR 1643072

[MJ] MoRITA, Y., \& JIMBO, S. Ordinary differential equations (ODE's) on inertial manifolds for reaction-diffusion systems in a singularly perturbed domain with several thin channels. J. Dynam. Differential Equations 4 (1992), 65-93. Zbl 0760.35026 MR 1150398

[Mo] Mosco, U. Convergence of convex sets and of solutions of variational inequalities. Adv. Math. 3 (1969), 510-585. Zbl 0192.49101 MR 0298508

[OPP] De Oliveira, L. A., Pereira, A. L., \& Pereira, M. C. Continuity of attractors for a reactiondiffusion problem with respect to variations of the domain. Electron. J. Differential Equations 2005, no. 100, 18 pp. Zbl 1176.35040 MR 2162261

[V] Vegas, J. M. Bifurcations caused by perturbing the domain in an elliptic equation. J. Differential Equations 48 (1983), 189-226. Zbl 0465.35075 MR 0696867

[VR] Vollmayr-Lee, B. P., \& Rutenberg, A. D. Fast and accurate coarsening simulation with an unconditionally stable time step. Phys. Rev. E 68 (2003), 066703, 13 pp.

[Z] ZeIdler, E. Nonlinear Functional Analysis and its Applications, Vol. I. Springer (1993). Zbl 0794.47033 MR 0816732 